\title{
Erratum: Distinct metabolomic signatures are associated with longevity in humans
}

Susan Cheng, Martin G. Larson, Elizabeth L. McCabe, Joanne M. Murabito, Eugene P. Rhee, Jennifer E. Ho, Paul F. Jacques, Anahita Ghorbani, Martin Magnusson, Amanda L. Souza, Amy A. Deik, Kerry A. Pierce, Kevin Bullock, Christopher J. O'Donnell, Olle Melander, Clary B. Clish, Ramachandran S. Vasan, Robert E. Gerszten \& Thomas J. Wang

Nature Communications 6:6791 doi: 10.1038/ncomms7791 (2015); Published 13 Apr 2015; Updated 21 May 2015

In Fig. 1c,d of this Article, the $y$ axis labels were inadvertently changed from 'Log isocitrate' to 'Log aconitase' during the production process. The correct version of Fig. 1 appears below.
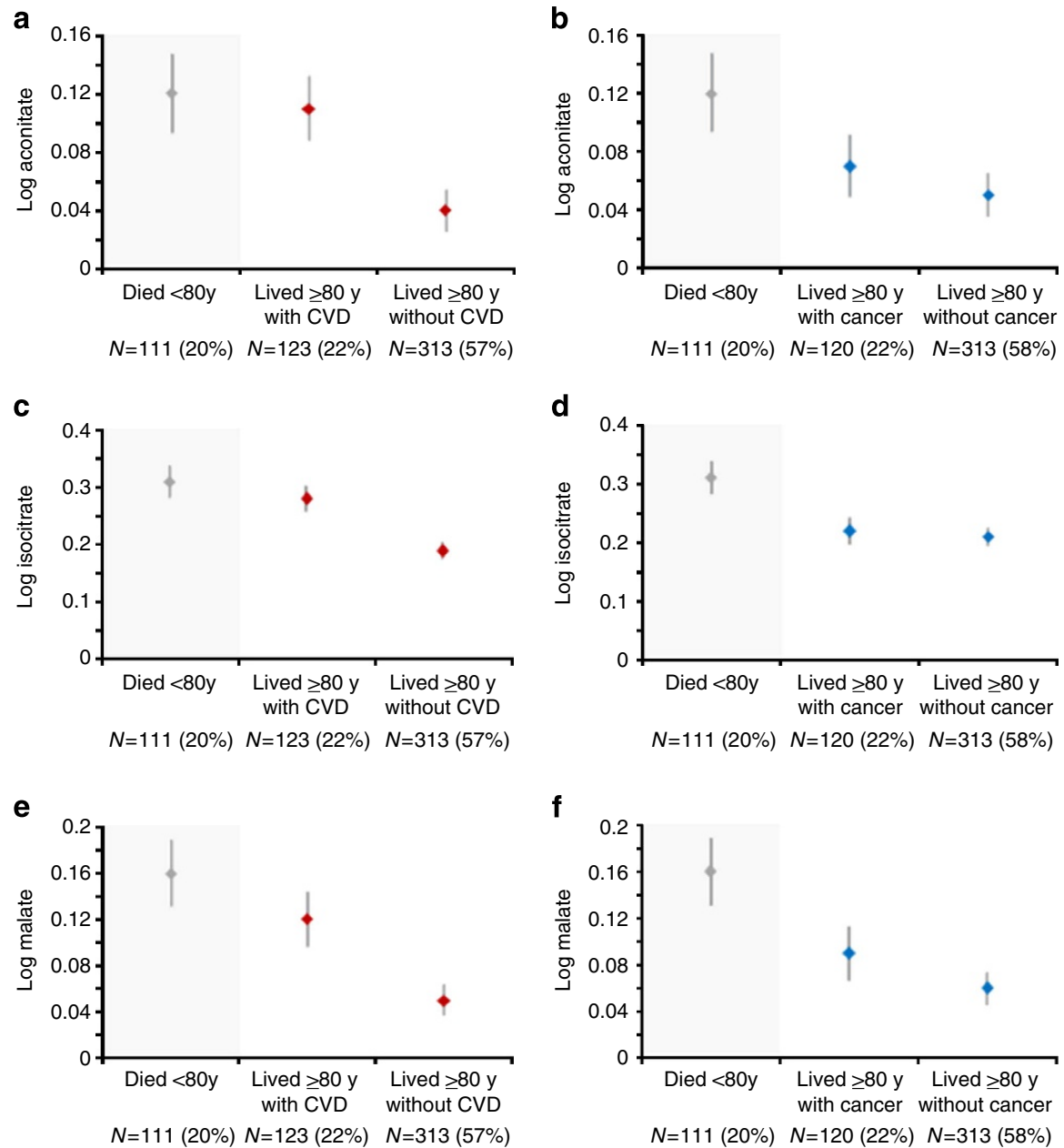

Figure 1 